\title{
Transplantation of adipose-derived mesenchymal stem cells attenuates pulmonary fibrosis of silicosis via anti-inflammatory and anti-apoptosis effects in rats
}

Shangya Chen ${ }^{1}$, Guanqun Cui ${ }^{2}$, Cheng Peng ${ }^{1,3^{*}}$, Martin F. Lavin ${ }^{1,4}$, Xiaoying Sun ${ }^{6}$, Enguo Zhang ${ }^{1,5}$, Ye Yang ${ }^{1,5}$, Yingjun Guan ${ }^{1}$, Zhongjun $\mathrm{Du}^{1 *}$ and Hua Shao ${ }^{1 *}$

\begin{abstract}
Background: Silicosis has been topping the list of high-incidence occupational diseases in developing countries and cannot be completely cured. Recent advances in stem cell research have made possible the treatment of various diseases including lung fibrosis. The application of stem cell therapy in occupational diseases, in particular the use of adipose-derived mesenchymal stem cells (AD-MSCs) in treatment of silicosis, has not yet been reported. The aim of the study is to explore the intervening effect of silica-induced lung fibrosis in rats.

Methods: In this study, we investigated the anti-pulmonary fibrosis effects of the transplantation of AD-MSCs in rats in which lung fibrosis was induced by oral tracheal intubation with silica suspension. Twenty rats were divided into four groups: control group $(n=5)$, exposure group $(n=5)$, vehicle group $(n=5)$ and treatment group $(n=5)$. AD-MSCs were given to rats after exposure to silica for $24 \mathrm{~h}$. Twenty-eight days after AD-MSC transplantation, we examined the organ coefficient, inflammatory cytokines, apoptosis, pathological and fibrotic changes in lung tissue.

Results: Results showed that exposure to silica for 28 days induced an increase of the lung coefficient with significant pulmonary fibrosis. Treatment with AD-MSC transplantation led to a remissive effect on pulmonary fibrosis. We found that after AD-MSC transplantation the inflammatory response decreased and Caspase-3 protein expression significantly decreased with a significant increase of the Bcl-2/Bax ratio.
\end{abstract}

Conclusions: Anti-inflammatory and anti-apoptosis of AD-MSCs may play important roles in their anti-pulmonary fibrosis effect. Our data suggest that transplantation of AD-MSCs holds promise for potential interference in the formation of silicosis through regulating inflammatory and apoptotic processes.

Keywords: Adipose-derived mesenchymal stem cells, Silicosis, Pulmonary fibrosis, Animal model, Transplantation, Cell therapy

\footnotetext{
*Correspondence: c.peng@uq.edu.au; duzj1981@163.com;

chinashaohua5888@163.com

'Department of Toxicology, Shandong Academy of Occupational Health and

Occupational Medicine, Shandong Academy of Medical Sciences, Jinan,

Shandong, People's Republic of China

Full list of author information is available at the end of the article
} 


\section{Background}

Silicosis refers to long-term inhalation and retention of silica dust in the lungs, mainly due to occupational exposure. The silicosis process involves the formation of silicon nodules, diffusion of pulmonary fibrosis and resultant diffused fibrotic pneumoconiosis [1]. Pathological characteristics of silicosis include alveolar epithelial cell injury and the formation of pulmonary fibrosis, but the mechanisms of silicosis are not fully clear. It is believed that inflammatory-related and apoptosis-related mechanisms play important roles in lung injury induced by silica dust and intervening/therapeutic routes targeting these pathways have been explored. For example, extensive attempts have been made to promote the treatment of silicosis through repairing injured cells but limited effects have been obtained. The use of stem cells as transplant tissue shows particular promise since stem cells have shown potency to repair damaged pulmonary tissue by replacing the endogenous damaged cells through cell regeneration and changing the microenvironment [2]. Recently, much progress has been made in understanding the mechanisms of mesenchymal stem cells (MSCs) in the aspects of regulating immunity and tissue remodeling in animal models of lung fibrosis [3]. In lung tissue rescue and repair, adult MSCs have been proposed to be a valuable therapeutic option due to their availability, immunomodulatory effects and anti-apoptotic and antiinflammatory properties [4].

As mesodermal-derived cells, bone marrow-derived mesenchymal stem cells (BM-MSCs) are primarily obtained through bone marrow aspiration. Mature adipose tissue collection can help extract primary AD-MSCs to obtain autologous AD-MSCs. Later, these mesodermalderived cells were proved to be pluripotent stem cells with multilineage differentiation potential and horizontal differentiation ability [5]. AD-MSCs have similar biological characteristics, immunosuppressive effects and multilineage differentiation potential to BM-MSCs which can differentiate into adipocytes, osteocytes, chondrocytes, myocytes and neural precursor cells [6-8]. However, because of the low content of BM-MSCs in bone marrow, prolonged time and complicated procedures for cell collection, culture and purification are required before transplantation. In contrast, AD-MSCs are plentiful in adipose tissue which is easy to access. To obtain the same amount of MSCs through bone marrow aspiration, the donor may only need to provide a smaller amount of adipose tissue, with relatively less pain and feasibility. These factors ensure AD-MSCs have a wider range of applications in stem cell treatment.

In recent years, a number of studies have tried to use MSCs for treatment of pulmonary injury. BM-MSCs transfected with HGF have been reported to be effective in improving pulmonary fibrosis in patients with silicosis. Researchers transfused autologous BM-MSCsHGF into silicosis patients, which reduced pulmonary small nodules significantly, with pulmonary function and inflammation of patients gradually ameliorated [9]. MSCs-HGF may be a potential treatment option for pulmonary injury. AD-MSC transplantation was also found to promote angiogenesis in the injured lung tissue by enhancing the expression of HGF [10]. The mechanism of action of MSCs in pulmonary diseases is thought to include regulating inflammation and promoting angiogenesis [11-14], and is affected by the quality of MSCs used [15-18]. AD-MSCs have been shown to be effective in repairing and regenerating lung tissue [19-21], including ameliorating idiopathic pulmonary fibrosis [22]. A recent study found that AD-MSCs can mitigate bleomycininduced lung fibrosis in aged mice [23]. However, silicosis-induced pulmonary fibrosis is different from bleomycin-induced pulmonary fibrosis in etiology. So far, there are currently no reported studies on AD-MSC treatment of silica-induced pulmonary fibrosis. Therefore, the aim of this study is to explore the preventive effect and mechanism of AD-MSC treatment for silicosis. To this end, we cultivated AD-MSCs for transplantation in rats with oral tracheal intubation with silica suspension and evaluated organ coefficients, pathological and fibrotic changes in lung tissue and inflammatory cytokines in lung tissue, and measured expression of the protein involved in the mitochondria-associated apoptotic pathway. Data from the study will provide evidence for the effects of AD-MSCs on silica-induced fibrosis and helpful information in the application of AD-MSCs for therapeutic purposes in the future.

\section{Methods}

Experimental animals and experimental design

Male Sprague Dawley (SD) rats weighting 180-200 g were purchased from Beijing Vital River Laboratory Animal Technology Co. Ltd (certificate No. SCXK (Jing) 2012-0001) and maintained under standard housing conditions (temperature $18-24{ }^{\circ} \mathrm{C}$; relative humidity 45 $60 \%$; light and dark cycle $12 \mathrm{~h}: 12 \mathrm{~h}$ ). Food and water were provided ad libitum. All animals were treated according to the protocols evaluated and approved by the experimental animal ethical committee of Shandong Academy of Medical Sciences.

Animal treatment and experimental design are shown in Fig. 1. Twenty adult male SD rats were randomly divided into four groups: control group $(n=5)$, which were normally fed; exposure group $(n=5)$, which were exposed to silica; vehicle group, $(n=5)$, in which DMEM culture medium was administered by intravenous injection $24 \mathrm{~h}$ after silica exposure; and treatment group $(n=5)$, which received $5 \times 10^{5} \mathrm{AD}-\mathrm{MSC}$ (suspended in DMEM culture medium) by intravenous injection $24 \mathrm{~h}$ after exposure to 


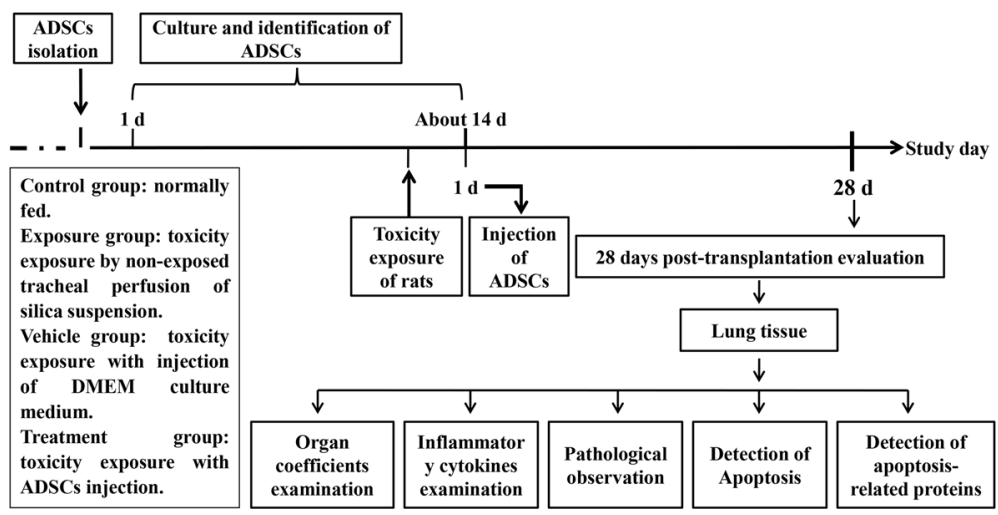

Fig. 1 Animal experiment design for anti-apoptotic effects of transplantation of AD-MSCs in adult rats. ADSC adipose-derived stem cell, d days, DMEM Dulbecco's modified Eagle's medium

silica. On the 28th day after transplantation, the animals from each group were evaluated for different parameters (Fig. 1).

\section{Culture and identification of stem cells}

AD-MSCs from adipose tissue of 4-week-old healthy male adult SD rats $(n=3)$ were isolated and cultured according to a previous report with slight modifications [24]. Briefly, the SD rats were sacrificed by $3 \%$ pentobarbital sodium anesthetic overdose. After being placed in $75 \%$ ethanol for about $5 \mathrm{~min}$, rats were dissected on a super-clean bench. The abdominal skin was cut along the midline of the abdomen until adipose tissue of the groin was exposed. Adipose tissue of the groin was collected and washed three times with phosphate buffered solution (PBS) containing $100 \mathrm{IU} / \mathrm{ml}$ penicillin and $100 \mu \mathrm{g} / \mathrm{ml}$ streptomycin (ThermoFisher Scientific, USA). After being transferred into a dry Petri dish, the tissue was cut quickly by sterilized ophthalmic scissors for $10 \mathrm{~min}$. The tissue pieces were collected and digested with collagenase dissolved in Dulbecco's modified Eagle's medium (DMEM) (with 5\% BSA, $5 \mathrm{mg} / \mathrm{ml}$ ) (ThermoFisher Scientific, USA) at $37{ }^{\circ} \mathrm{C}$ for $60 \mathrm{~min}$. After centrifugation at $1000 \mathrm{rpm}$ (Sorvall ST8R; ThermoFisher Scientific, USA), the cell pellet was suspended in complete medium (DMEM with 10\% embryonic stem cell-qualified fetal bovine serum, $100 \mathrm{IU} / \mathrm{ml}$ penicillin and $100 \mu \mathrm{g} / \mathrm{ml}$ streptomycin) (Biological Industries, USA). The cell suspension was filtered through a cell filter with $70-\mu \mathrm{m}$ pores. A cell suspension with $10^{4}$ cells/ $\mathrm{ml}$ was obtained and incubated in $\mathrm{CO}_{2}$ incubator (Labserv CO-150; ThermoFisher Scientific, USA) after being washed with DMEM and centrifuged as already described. The cells were observed daily and photographs were taken under inverted microscope (IX70; Olympus, Japan).

AD-MSCs were identified and selected by flow cytometry (FCM) with CD44, CD45, CD90, CD73 and CD11b antibodies. After being subcultured to the third generation, AD-MSCs at $80 \%$ confluence were washed twice with PBS followed by digestion with $0.25 \%$ trypsinEDTA (ThermoFisher Scientific, USA). The cells were then centrifuged at $1000 \mathrm{rpm}$ and washed with PBS. After incubation with antibodies and their isotype controls (1:100) (Becton Dickinson and Co., USA) at $4{ }^{\circ} \mathrm{C}$ for $30 \mathrm{~min}$, the cells were flowed through the cytometer at about 1000 cells per second. Results of FCM were analyzed by FlowJo software (FlowJo, LLC, USA).

AD-MSCs were also identified by adipogenic, osteogenic and chondrogenic differentiation. After being subcultured to the third generation, AD-MSCs at $80 \%$ confluence were induced in adipogenesis, osteogenic and chondrogenic differentiation complete medium (using adipogenesis, osteogenic and chondrogenic differentiation kits from ThermoFisher Scientific, USA). After induction for 21 days, cells were fixed with $4 \%$ paraformaldehyde for about $20 \mathrm{~min}$. The intracellular lipids accumulated in the induced AD-MSCs in adipogenic cultures were stained with Oil Red O (Sigma-Aldrich, USA). The mineralized osteogenic cultures were stained with Alizarin Red S (Sigma-Aldrich, USA) to detect calcium deposition. The mucopolysaccharide accumulated in chondrogenic cultures were stained with Toluidine Blue (Sigma-Aldrich, USA). Photographs were taken under the inverted microscope.

\section{Silica-induced silicosis in rats}

We improved the method of oral tracheal intubation with silica suspension to induce silicosis in rats. The micron-sized silica (approximately $80 \%$ between 1 and 5 $\mu \mathrm{m}$; Sigma-Aldrich, USA) was autoclaved and made into a suspension at $50 \mathrm{mg} / \mathrm{ml}$ in normal saline. Rats were fixed on the operating table after $3 \%$ pentobarbital sodium anesthesia. Incisors of rat were pulled down by surgical suture and the tongue of the rat was pulled out by sterile forceps with the light of exceeded luminosity lamp-house toward the neck of the rat. The glottis was the bright spot opening and closing with breath which 
could be seen through the mouth. Then we wiped the mucus near the glottis with a cotton swab. When the glottis was open, the indwelling venous needle was inserted into the trachea. Silica suspension $(1 \mathrm{ml})$ was injected quickly into the cannula. Then the rat was gently shaken for about 5 min to distribute the suspension uniformly in the lungs. The rats in the control group were perfused with $1.0 \mathrm{ml}$ of sterile normal saline.

\section{Transplantation procedure}

AD-MSCs were resuspended at a concentration of $5 \times 10^{5}$ cells $/ \mathrm{ml}$ for transplantation which was performed on rats exposed to silica for $24 \mathrm{~h}$. The cell dosage was $1 \times 10^{6}$ cells $/ \mathrm{kg}$. Briefly, rats were anesthetized with $3 \%$ pentobarbital sodium. The rat tail was sterilized by $75 \%$ ethanol. Then $1 \mathrm{ml}$ of cell suspension with $5 \times 10^{5}$ cells/ $\mathrm{ml}$ was injected over 2 min using a disposable vein infusion needle which was attached to a disposable syringe of $1 \mathrm{ml}$. In order to minimize cell leakage, the needle was left in place for $2 \mathrm{~min}$ after injection before withdrawal. The vehicle group had DMEM culture medium "transplanted" in the same way.

\section{Inflammatory cytokine measurement}

TNF- $\alpha$, IL-1 $\beta$, IL- 6 and IL-10 were detected by ELISA using different ELISA kits (ThermoFisher Scientific, USA) according to the manufacturer's protocols. Briefly, rats from each group were euthanized 28 days post transplantation. The lungs of rats were rapidly removed, weighed and frozen at $-80{ }^{\circ} \mathrm{C}$ for further experiment. After being diluted, detection A solution was added into the plate at $100 \mu \mathrm{l}$ per well and placed at $37^{\circ} \mathrm{C}$ for $1 \mathrm{~h}$. After the plate was washed three times, detection $\mathrm{B}$ solution was added as detection $\mathrm{A}$ and placed at $37^{\circ} \mathrm{C}$ for $30 \mathrm{~min}$. The plate was then washed five times, and $3,3^{\prime}, 5,5^{\prime}$-tetramethylbenzidine (TMB) was added at $90 \mu \mathrm{l}$ per well and placed at $37{ }^{\circ} \mathrm{C}$ for $20 \mathrm{~min}$. Finally, sulfuric acid was added at $50 \mu \mathrm{l}$ per well as the termination solution. The optical density (OD) of each well of a 96-well plate at $450 \mathrm{~nm}$ was analyzed using a microplate reader (SpectraMax 190; Molecular Devices, USA).

\section{Histopathological and fibrotic examination}

Rats were deeply anesthetized with $3 \%$ pentobarbital sodium 28 days post transplantation. The body weight was measured and the heart, liver, spleen and lungs of rats were isolated and excised with a razor blade and weighed, and the organ coefficient (the ratio of the organ weight to the body weight) was calculated. The organ coefficient is an important indicator to evaluate toxic effects of test substances in toxicology research. Then, the lungs of rats were collected and some parts were fixed in $4 \%(\mathrm{v} / \mathrm{v})$ paraformaldehyde. After embedding in paraffin blocks, tissues were sectioned into $5 \mu \mathrm{m}$ slice, and then mounted onto glass slides. Tissues on the glass slides were stained with hematoxylin and eosin (H\&E) for histopathological evaluation, and stained with Masson's trichrome stain for fibrotic examination. Photographs were taken using an optical microscope (BX51; Olympus, Japan). The slides were examined by pathologists who were blinded to the identity and analysis of pathology sections. The results were peer-reviewed by other certified veterinary pathologists. Fibrotic changes were quantified by modified Ashcroft score with a grade of $0-8$ to represent alveolar structure from normal lung to total fibrous obliteration of lung fields [25].

\section{Apoptosis detection}

The lungs of rats were processed as already described for terminal deoxynucleotide transferase (TdT)-mediated dUTP nick end labeling (TUNEL) using an in-situ cell death detection kit (Roche, Switzerland) according to the manufacturer's instructions. Briefly, lung slides of each group were fixed with $4 \%$ paraformaldehyde in 0.1 $\mathrm{M}$ phosphate buffer ( $\mathrm{pH}$ 7.4). After incubation with proteinase $\mathrm{K}(100 \mu \mathrm{g} / \mathrm{ml})$, sections were washed with PBS, and permeabilized with $0.1 \%$ Triton $\mathrm{X}-100$. The sections were then washed with PBS twice and incubated in TUNEL reaction mixture. After sections were rinsed again, the converter-POD was used to visualize sections with $0.02 \%$ 3,3'-diaminobenzidine (DAB). After counterstaining with Mayer's hematoxylin, sections were mounted on the slides which were gelatin-coated and placed at room temperature overnight to be air-dried. Positive results were brown-stained nucleus. An apoptotic index was evaluated to analyze differences between groups in apoptosis detection. The apoptosis index was determined from 10 blindly selected high-power fields for each slice by counting the number of positive cells in 500-1000 cells per field to calculate the percentage of positive cells.

\section{Western blot assay}

We used western blot analysis to detect the expression of Bax, Bcl-2 and Caspase- 3 proteins in lung tissue of rats from each group. The lungs were processed as already described and lung tissues were homogenized for protein extraction. After being resuspended in homogenization buffer, the tissues were centrifuged for $10 \mathrm{~min}\left(12,000 \mathrm{rpm}, 4{ }^{\circ} \mathrm{C}\right)$. Supernatants were collected as protein suspension. SDS-PAGE (12\% resolving gels at $120 \mathrm{~V}, 5 \%$ stacking gels at $75 \mathrm{~V}$ ) was used to separate protein samples. Then protein samples were transferred to PVDF membranes (200 $\mathrm{mA}, 1 \mathrm{~h}$ ). The membranes were blocked with $5 \%$ nonfat dry milk followed by incubation with different primary antibodies and $\beta$-actin (Santa Cruz, USA) overnight at $4{ }^{\circ} \mathrm{C}$. After being washed with TBST, the membranes were incubated with 
secondary antibodies (Santa Cruz, USA) at 1:1000 dilutions for $1.5 \mathrm{~h}$ at room temperature. After being washed with TBST, the membranes were treated with enhanced chemiluminescence (ECL detection kit; Pierce) and exposed to the Odyssey CLx near-infrared fluorescence imaging system (LI-COR, USA). Results were quantified by AlphaEaseFC software (Alpha Innotech, USA). We performed experiments in triplicate to ensure reproducibility.

\section{Statistical analysis}

Data are presented as means \pm SD. Statistical analysis was performed using one-way analysis of variance (ANOVA). Data for each group were compared with other groups by least-significant difference (LSD) for significance. All statistical analyses used SPSS software (IBM SPSS Statistics 20.0, USA). Results were considered statistically significant at $p<0.05$.

\section{Results}

\section{Culture and identification of AD-MSCs}

Primary cells were mainly spindle-shaped and partially polygonal, and showed rapid proliferation in the first 3 days. With the cell expansion progressed, most cells were long spindle-shaped and fibroblast-like with the number of hybrid cells decreased significantly, and the proliferation rate of AD-MSCs decreased gradually. After expansion to the third generation, the cells were long spindle-shaped and evenly distributed, and cells were whirlpool-like or chrysanthemum-like aggregated at a density of $100 \%$. The cell morphology was more consistent with a faster growth rate. Flow cytometry measurement of cells at the third generation showed that expression of CD44, CD73 and CD90 was positive (99.15\%, 96.30\% and 99.88\%), with negative expression of CD11b and CD45 (0.26\% and 0.82\%). Meanwhile, adipogenic, osteogenic and chondrogenic differentiation of cells at the third generation all succeeded. Thus, these results confirmed that the vast majority of cells in the third generation were AD-MSCs (Fig. 2).

\section{Organ coefficients of rats}

The organ coefficient is considered a commonly used indicator of animal status. After rats were sacrificed, organs were removed and rinsed with normal saline. The organs were then blotted dry on filter paper and immediately weighed. The ratio of organ weight/body weight was considered the organ coefficient. There was no significant difference among the heart coefficient of rats in different groups. Compared to the control group, the lung coefficients of rats significantly increased in the treatment group, exposure group and vehicle group $(p<0.05)$ (Table 1$)$.

\section{AD-MSC transplantation recovers the silica-induced inflammatory cytokines}

Inflammatory cytokines of lung tissue were evaluated after rats were sacrificed (Table 2). Compared to the control group, the TNF- $\alpha$, IL-1 $\beta$, IL- 6 and IL-10 levels of rats significantly increased in rats in the exposure group and vehicle group $(p<0.05)$. There was no significant difference between the exposure and vehicle groups for each inflammatory cytokine. Notably, the TNF- $\alpha$, IL$1 \beta$, IL- 6 and IL-10 levels in the treatment group significantly decreased compared to the vehicle group $(p<0$. $05)$. The expression levels of TNF- $\alpha$, IL- 6 and IL-10 significantly decreased in the treatment group, with the IL$1 \beta$ level decreased compared to the exposure group $(p<$ 0.05 ). There was also a significant increase of IL- $1 \beta$ in rats from the treatment group compared to the control group.

\section{AD-MSCs mitigate the lung pathology changes by silica}

The alveolar structure of rats from the control group was intact with no inflammatory cell infiltration or fibrosis, as shown in Fig. $3 \mathrm{Aa}_{1}, \mathrm{Bb}_{1}$. Lung alveolar structures in rats from the exposure and vehicle groups were severely destroyed with a large amount of inflammatory cell infiltration, phagocytic cells and silicon nodules (Fig. $3 \mathrm{Aa}_{2}, \mathrm{a}_{3}$ ). Telangiectasia, interstitial lymphocytic infiltration, alveolar epithelium disarrangement and pulmonary vascular wall thickening were observed in rats from the exposure and vehicle groups (Fig. $3 \mathrm{Bb}_{2}, \mathrm{~b}_{3}$ ). Alveolar structure destruction, scattered fusion of silicon nodules, inflammatory cells and lymphocytes were significantly reduced in the treatment group compared with the exposure group (Fig. $3 \mathrm{Aa}_{4}, \mathrm{Bb}_{4}$ ). Collagen fibers, mucus and cartilage were stained blue, while cytoplasm, muscle, cellulose and glial were stained red in Masson staining. After Masson staining, normal collagen fiber stent could be observed in the lung tissue of the control group (Fig. $4 \mathrm{Aa}_{1}, \mathrm{Bb}_{1}$ ). There was a large amount of collagen fibers deposited in the pulmonary mesenchyme, especially around bronchi and vessels in the exposure group and vehicle group (Fig. $4 \mathrm{Aa}_{2}, \mathrm{a}_{3}, \mathrm{Bb}_{2}, \mathrm{~b}_{3}$ ). Collagen fiber deposition could also be found in the treatment group, but the area of deposition was decreased compared to the exposure group or vehicle group (Fig. $4 \mathrm{Aa}_{4}, \mathrm{Bb}_{4}$ ). The result of modified Ashcroft score evaluation was consistent with the result of the slice observation, as shown in Fig. 5.

\section{AD-MSCs reduced the silica-induced cellular apoptosis}

We used the TUNEL method to detect the apoptosis level in lung tissues (Fig. 6). TUNEL-positive cells were observed to distribute in rats of the exposure, vehicle and treatment groups 28 days after transplantation. The apoptosis index showed much difference among the control group and the three other groups. An increased apoptosis index in the exposure, vehicle and treatment groups was 


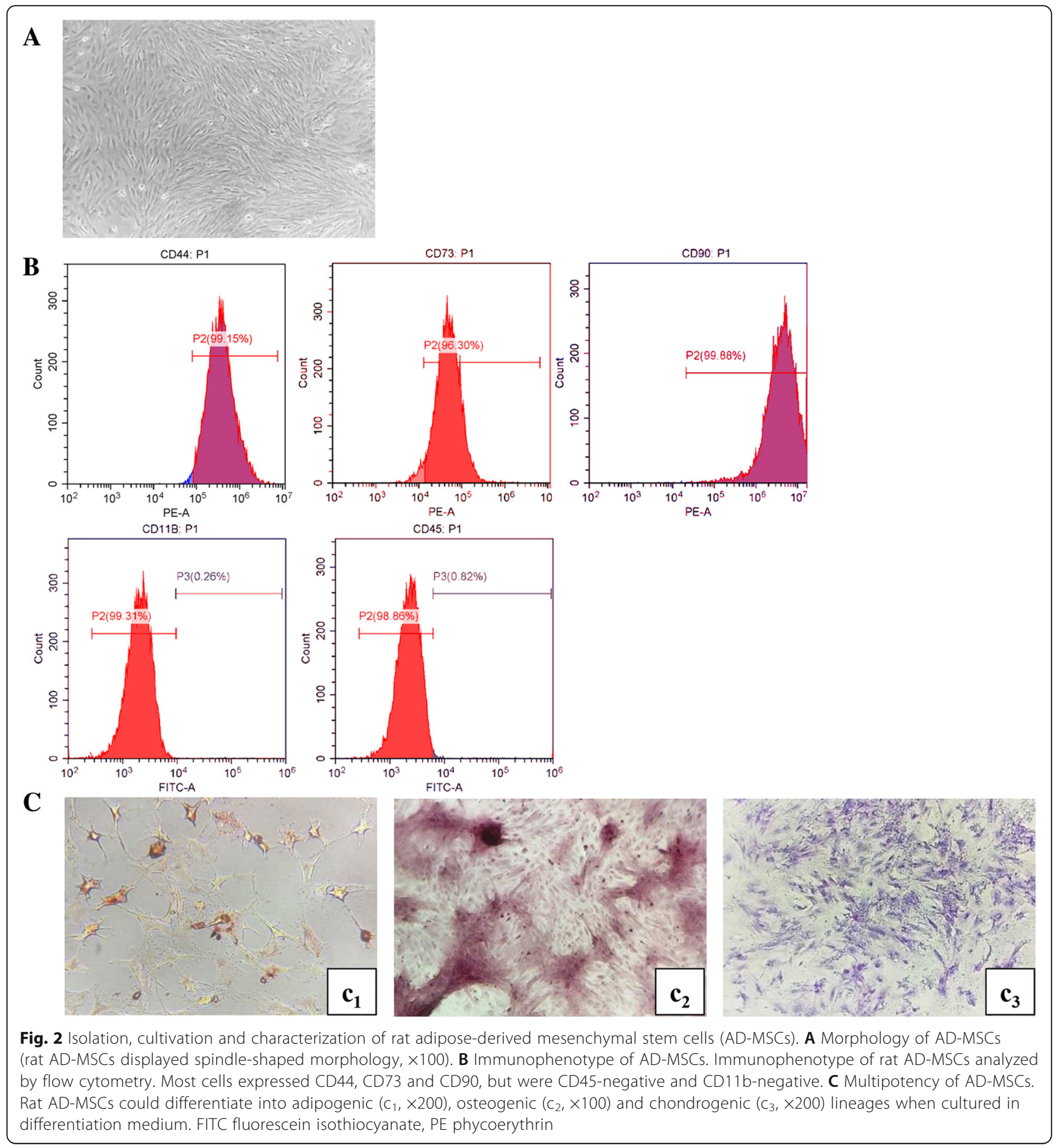

Table 1 Organ coefficients of rats after surgery (mean $\pm \mathrm{SD}, n=5$ )

\begin{tabular}{lll}
\hline Group & Lung coefficient (\%) & Heart coefficient (\%) \\
\hline Control group & $0.57 \pm 0.04$ & $0.32 \pm 0.05$ \\
Exposure group & $0.90 \pm 0.08^{\mathrm{a}}$ & $0.31 \pm 0.02$ \\
Vehicle group & $0.89 \pm 0.11^{\mathrm{a}}$ & $0.30 \pm 0.01$ \\
Treatment group & $0.86 \pm 0.24^{\mathrm{a}}$ & $0.31 \pm 0.02$ \\
\hline
\end{tabular}

${ }^{a} p<0.05$, vs control group observed compared with control group. The apoptosis index in the treatment group was significantly decreased compared with that in the exposure group $(p<0.05)$. No significant difference of the apoptosis index was seen between the vehicle group and the exposure group.

\section{Expressions of $\mathrm{Bax}, \mathrm{Bcl}-2$ and Caspase-3}

We found that silica exposure led to upregulation of Caspase-3 protein and downregulation of $\mathrm{Bax}$ and $\mathrm{Bcl}-2$. 
Table 2 Inflammatory cytokines in lungs of rats after surgery (mean $\pm S D, n=5$ )

\begin{tabular}{lllll}
\hline Group & TNF-a $(\mathrm{pg} / \mathrm{ml})$ & $\mathrm{IL}-1 \beta(\mathrm{pg} / \mathrm{ml})$ & $\mathrm{LL}-6(\mathrm{pg} / \mathrm{ml})$ & $\mathrm{IL}-10(\mathrm{pg} / \mathrm{ml})$ \\
\hline Control group & $31.95 \pm 8.06$ & $443.18 \pm 376.22$ & $153.00 \pm 106.11$ & $165.63 \pm 32.09$ \\
Exposure group & $108.44 \pm 16.90^{\mathrm{a}}$ & $1355.97 \pm 397.43^{\mathrm{a}}$ & $521.30 \pm 193.05^{\mathrm{a}}$ & $334.45 \pm 77.70^{\mathrm{a}}$ \\
Vehicle group & $108.41 \pm 15.10^{\mathrm{a}}$ & $1600.92 \pm 234.88^{\mathrm{a}}$ & $569.65 \pm 126.07^{\mathrm{a}}$ & $389.74 \pm 53.36^{\mathrm{a}}$ \\
Treatment group & $43.07 \pm 8.33^{\mathrm{b}, \mathrm{c}}$ & $1018.24 \pm 102.88^{\mathrm{a}, \mathrm{c}}$ & $164.81 \pm 51.66^{\mathrm{b}, \mathrm{c}}$ & $180.81 \pm 38.44^{\mathrm{b}, \mathrm{c}}$ \\
\hline
\end{tabular}

IL interleukin, TNF tumor necrosis factor

${ }^{a} p<0.05$, vs control group

${ }^{\mathrm{b}} p<0.05$, vs exposure group

${ }^{c} p<0.05$, vs vehicle group

After oral tracheal intubation with silica suspension, expression of Caspase- 3 protein was significantly upregulated, while expression of $\mathrm{Bax}$ and $\mathrm{Bcl}-2$ proteins was significantly downregulated (Fig. 7). In rats with ADMSC transplantation, compared with those in the exposure and vehicle groups, Caspase- 3 protein significantly decreased while $\mathrm{Bax}$ and $\mathrm{Bcl}-2$ protein expression increased. Further analysis showed that the ratio of Bcl-2/ Bax in the exposure and vehicle groups was significantly decreased, and the $\mathrm{Bcl}-2 / \mathrm{Bax}$ ratio in the treatment group was significantly increased when compared with the exposure group and vehicle group.

\section{Discussion}

Recently, much attention has been paid to stem cell therapy in experimental silicosis since it appears to be susceptible to therapeutic intervention. Transplantation of BM-MSCs has been demonstrated to be a promising treatment for therapy of silicosis [26]. Clinical study has shown that transplantation of autologous BM-MSCs can effectively reduce pulmonary fibrosis with and improve lung function in patients with silicosis [24]. However, with current techniques, it is hard to obtain stem cells from bone marrow and this hinders the use of BMMSCs. In contrast, AD-MSCs have a wider range of sources and are more readily available. In addition, liposuction is more likely to be accepted by patients compared with bone marrow puncture in clinical practice. To take these advantages, we selected AD-MSCs for intervening in experimental silicosis. The effect of ADMSCs on silica-induced silicosis has not been reported. In this study, we successfully obtained AD-MSCs from adipose tissue of rats and cultured them in vitro. Primary AD-MSCs showed rapid proliferation in the first 3 days. After transfer to the third generation, the cells with a long spindle-shaped morphology were more uniform with a faster growth rate (Fig. 2). Then, we verified the cells by checking the expression of surface molecules and their adipogenic, osteogenic and chondrogenic differentiation ability (Fig. 2).

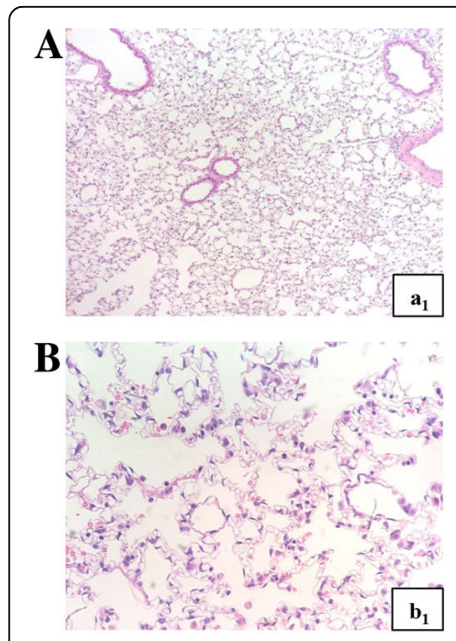

Control
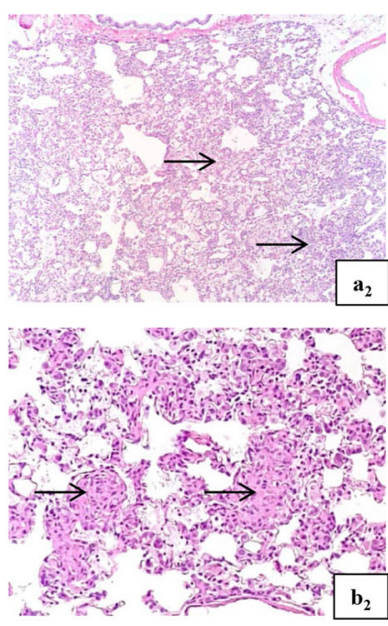

Exposure

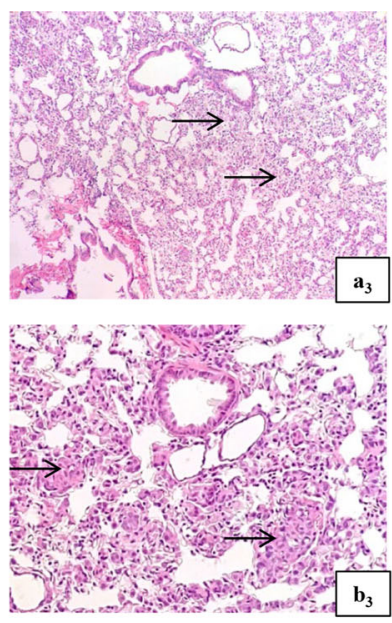

Vehicle

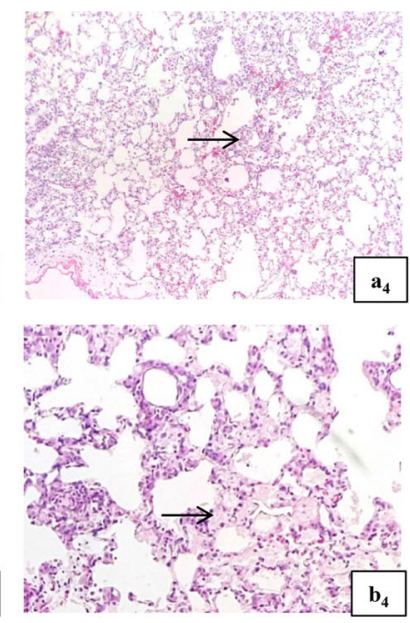

Treatment

Fig. 3 H\&E staining of lung tissue in each group. A $\times 100$; $\mathbf{B} \times 400 .\left(a_{1}, b_{1}\right)$ Control group, normal lung morphology: thin lined interalveolar septa with well-organized alveolar space. $\left(a_{2}, b_{2}\right)$ Exposure group, distorted lung morphologies: collapsed alveolar space with inflammatory exudates, wider and thickened interalveolar septa, initial formation of silicon nodules. $\left(a_{3}, b_{3}\right)$ Vehicle group, similar to exposure group. $\left(a_{4}, b_{4}\right)$ Treatment group, ameliorative lung morphology: lessoned collapsed alveolar space with inflammatory exudate, less widened and thickened alveolar septum, reduced pronounced nodules of silicon. Arrows point out pathological changes of lung tissue 


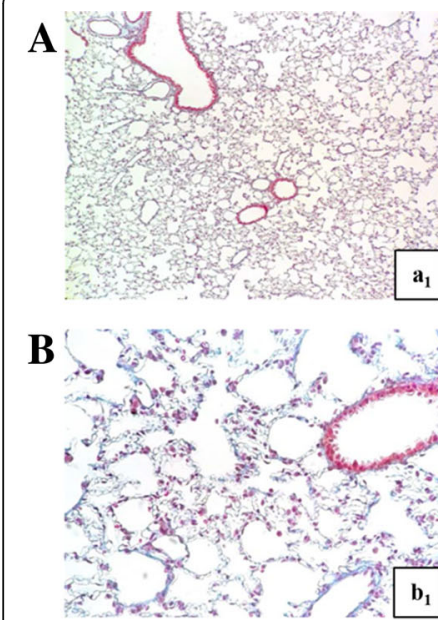

Control
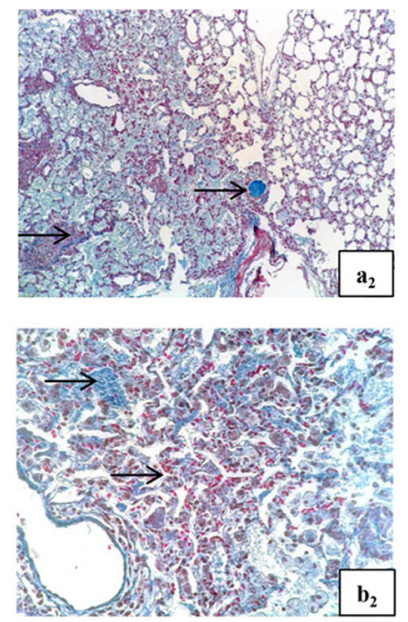

Exposure
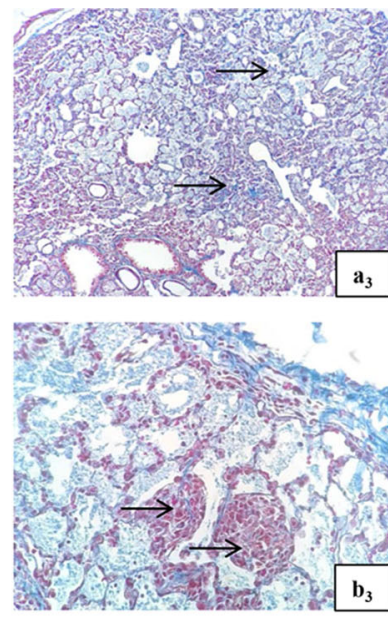

Vehicle
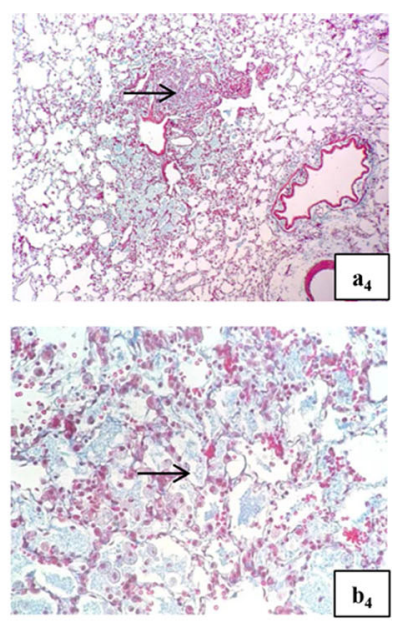

Treatment

Fig. 4 Masson staining of lung tissue in each group. $\mathbf{A} \times 100$; $\mathbf{B} \times 400 .\left(a_{1}, b_{1}\right)$ Control group, normal lung morphology: almost no deposition of collagen in lung parenchyma. $\left(a_{2}, b_{2}\right)$ Exposure group, distorted lung morphologies: collagen fiber agglomerates, initial formation of silicon nodules and dense accumulation of collagen. $\left(a_{3}, b_{3}\right)$ Vehicle group, similar to exposure group. $\left(a_{4}, b_{4}\right)$ Treatment group, ameliorative lung morphology: lung sections showing reduced collagen deposition, less alveolar thickening and reduced amounts of collagen. Arrows point out pathological changes of lung tissue

Pulmonary fibrosis is characterized by a certain degree of lung inflammation and abnormal tissue repair involving a complex network of cytokines. These events result in increased collagen gene expression and abnormal collagen deposition in the lungs that eventually produce fibroblast foci [27]. In order to observe the distribution of inflammatory cells and detect collagen deposition in lung interstitium, we used H\&E staining and Masson staining to evaluate differences in the progression of pulmonary fibrosis between groups. Rats from the exposure groups showed positive staining, which suggested the formation of lung fibrosis (Figs. 3 and 4). The quantitative score of pathological analysis of pulmonary fibrosis was then conducted by estimation of the Modified Ashcroft scale [25]. We found that inflammatory cells aggregated and collagen

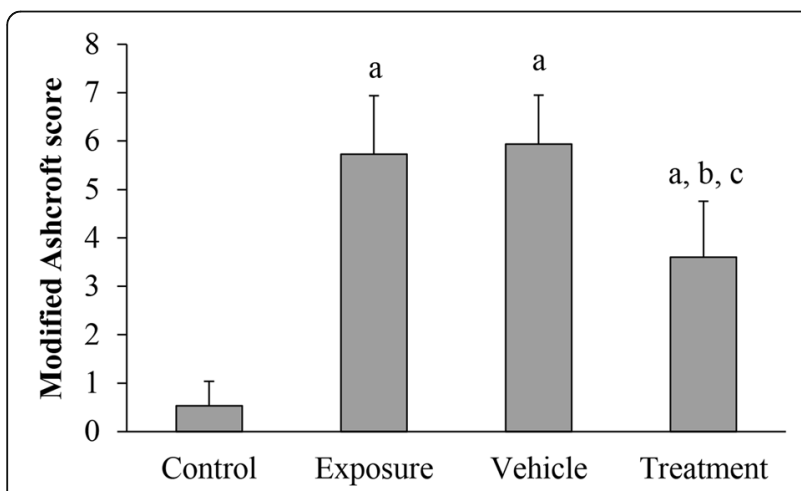

Fig. 5 Severity of pulmonary fibrosis evaluated by modified Ashcroft score. Presented as mean \pm SD $(n=5) .{ }^{a} p<0.05$, vs control group; ${ }^{\mathrm{b}} p<0.05$ vs exposure group; ${ }^{\mathrm{c}} p<0.05$ vs vehicle group was deposited in the lung, accompanied by severe distortion of pulmonary structure and large fibrous areas in rats exposed to silica suspension (Figs. 3 and 4). After ADMSC transplantation, decreased inflammatory cells and collagen deposition in the lung tissue, reduced damage to the lung structure and formation of small fibrous masses were found in the lung interstitium (Fig. 4). The results showed that early intervention of AD-MSCs could reduce inflammatory reaction and slow down the process of pulmonary fibrosis.

Researchers hypothesized that the inflammatory reaction mechanisms underlying MSC therapy of pulmonary fibrosis were mediated by paracrine signaling (Reference). The therapeutic effect of BM-MSCs was found to trigger IL-1RA secretion to suppress the upregulated IL-1 and TNF- $\alpha$ proteins to protect the lung against damage and fibrosis [26]. Consistent with several previous studies using fetal membrane-derived stem cells and preconditioned BM-MSC transplantations as bleomycin-induced pulmonary fibrosis therapies [27, 28], we demonstrated that AD-MSC transplantation can reduce the pulmonary inflammatory response of rats after oral tracheal intubation with silica suspension, indicating the migration and homing of AD-MSCs toward the lungs through intravenous infusion to affect the inflammatory response to silica exposure. Downregulated expression of TNF- $\alpha$, IL-1 $\beta$, IL- 6 and IL-10 proteins indicated that AD-MSCs can also protect the lungs from injury and fibrosis through an anti-inflammatory process, which was consistent with ample experimental evidence in MSC-treated pulmonary fibrosis [28-30]. 

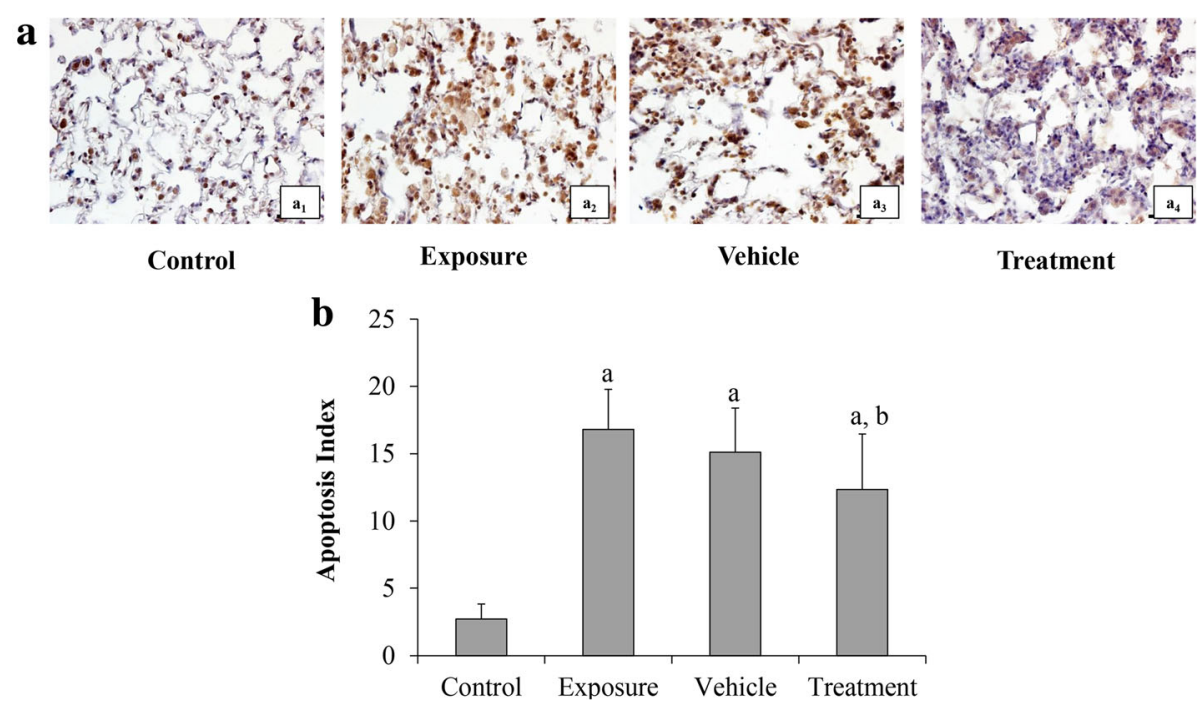

Fig. 6 Cellular apoptosis of lung tissue in each group (TUNEL staining). a $\times 400:\left(a_{1}\right)$ control group; $\left(a_{2}\right)$ exposure group; $\left(a_{3}\right)$ vehicle group; $\left(a_{4}\right)$ treatment group. $\mathbf{b}$ Apoptosis index of each group. ${ }^{a} p<0.05$ vs control group; ${ }^{b} p<0.05$ vs exposure group

Macrophages can stimulate epithelial cells by activating and releasing inflammatory cytokines TNF- $\alpha$, IL-1, IL-6 and IL-10. Activated epithelial cells can regulate local immunity by recruiting locally inhabiting immunocompetent cells to provide intercellular and intracellular communication through autocrine and paracrine signaling pathways [31]. IL-1 $\beta$ and TNF- $\alpha$ mainly activate the nuclear factor- $\mathrm{kB}$ pathway [32, 33], while IL-6 and IL-10 exert their complex actions through multiple pathways like the Janus kinase 2/signal transducers and activators of transcription 3 pathway and the p38 mitogenactivated protein kinase/extracellular signal-regulated kinase pathway $[34,35]$. TNF- $\alpha$, IL- $1 \beta$ and IL- 6 are regarded as proinflammatory cytokines, and IL-10 is
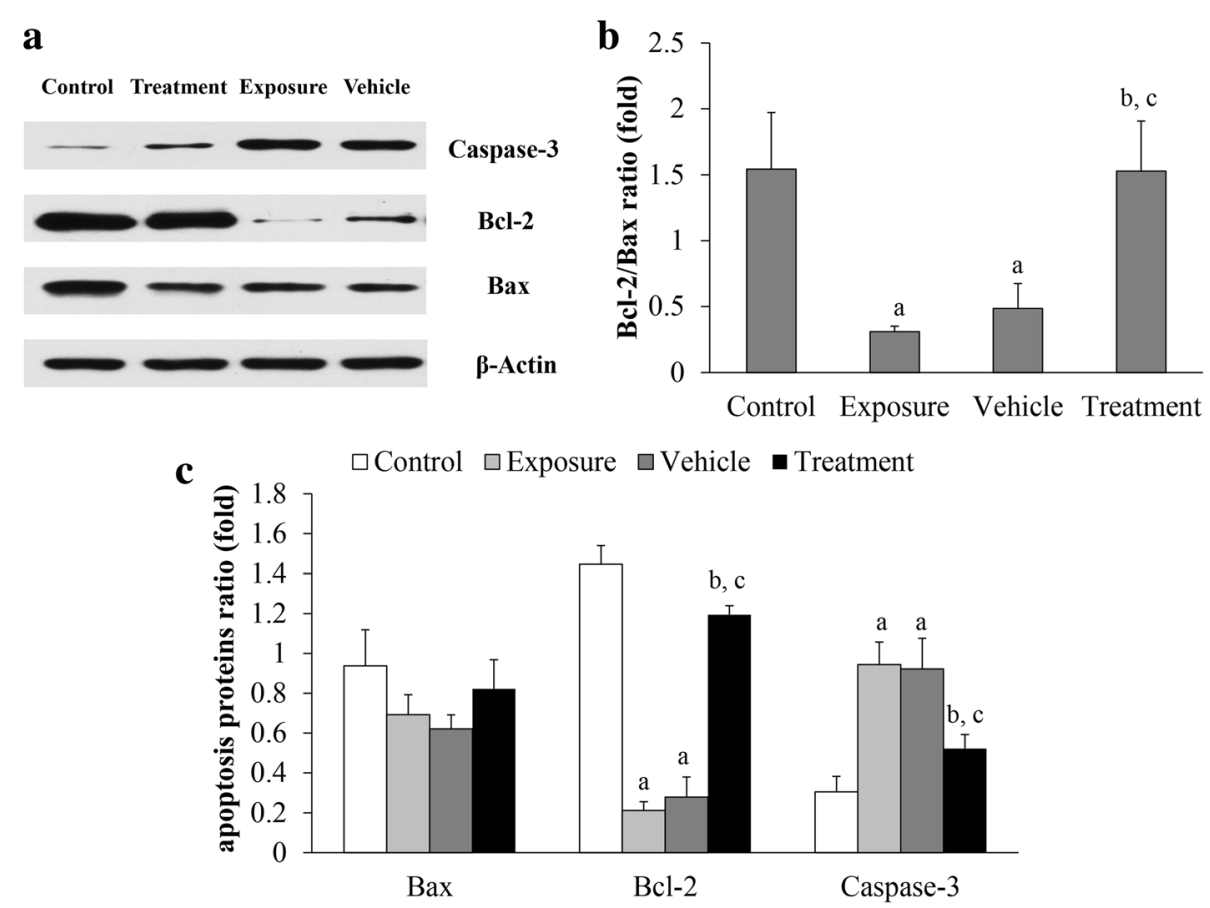

Fig. 7 Expression levels of $\mathrm{Bax}, \mathrm{BCl}-2$, Caspase-3 and $\beta$-actin proteins of lung tissue in rats 28 days after transplantation. a Western blot analysis of $\mathrm{Bax}, \mathrm{BCl}-2$, Caspase- 3 and $\beta$-actin proteins of lung tissue in rats. $\mathbf{b} \mathrm{BCl}-2 / \mathrm{Bax}$ ratio of lung tissue in each group. $\mathbf{c}$ Relative ratio of Bax, $\mathrm{BCl}-2$ and Caspase-3 protein of lung tissue in rats. ${ }^{a} p<0.05$ vs control group; ${ }^{b} p<0.05$ vs exposure group; ${ }^{c} p<0.05$ vs vehicle group 
generally considered to be an anti-inflammatory cytokine. It was also shown that overexpression of IL-10 aggravates silica-induced pulmonary inflammation and fibrosis in mice, which might relate to the promotion of Th2-type cytokine reaction and increased expression of IL-4 and IL-13 [32]. We found a downregulated expression of IL-10 at day 28 after transplantation, which might indicate the benefit of low expression of IL-10 in lung tissue during pulmonary fibrosis prohibition exerted by AD-MSC transplantation. In this regard, the effect of AD-MSC transplantation on pulmonary inflammatory factor release may directly alter the formation of pulmonary fibrosis through an anti-inflammatory pathway.

Apoptosis, inflammatory reaction and their cascade reactions can signify secondary damage after lung injury $[36,37]$. Therefore, we tested the anti-apoptotic effect of AD-MSCs in a rat silicosis model and found that the apoptosis index of lung cells in AD-MSC-treated rats was decreased compared with that of the exposure group and vehicle group. We also found that the apoptosis of pulmonary cells in rats exposed to silica involved regulation processing of $\mathrm{Bax}, \mathrm{Bcl}-2$ and Caspase-3, thus probably resulting in the apoptotic death of cells in lung tissue. The increase in Caspase family protease activity is associated with apoptosis, in which Caspase-3 plays a pivotal part in the apoptosis of bleomycin-induced pulmonary fibrosis [38]. Further, our data showed decreased apoptosis in the treatment group using TUNEL assay (Fig. 6). Thus, the results suggested that AD-MSC transplantation in rats exposed to silica suspension exerted an anti-apoptotic effect.

Anti-apoptotic protein $\mathrm{Bcl}-2$, pro-apoptotic protein Bax and Caspase-3 play an important role in the progression of apoptosis [39]. Bax regulates apoptosis by forming homologous dimers or heterodimers with $\mathrm{Bcl}-2$ to produce an apoptotic regulatory system. Owing to the stability of heterodimer $\mathrm{Bax}-\mathrm{Bcl}-2$ compared to $\mathrm{Bax}-$ $\mathrm{Bax}$, the ratio of $\mathrm{Bcl}-2 / \mathrm{Bax}$ regulates the occurrence of apoptosis and determines cell survival. Pulmonary cells are in a relatively stable proportion of pro-apoptotic protein Bax and anti-apoptotic protein Bcl-2 in physiological conditions, maintaining the steady-state balance of pulmonary structure and function. The Caspase family mainly exists in the form of zymogen in cells. When stimulated by the apoptotic signal, initiator Caspases are activated, and then the executioner Caspases. They induce apoptosis by decomposing substrate proteins. It has been shown that Caspase-3 may play an important role in apoptosis and has the effect of removing inhibition to mediate feedback amplification [40]. Therefore, we examined the expression of Bax, Bcl-2 and Caspase- 3 in apoptosis by western blot assay in order to investigate the mechanism of apoptosis suppression after AD-MSC transplantation in rats with oral tracheal intubation with silica suspension. The results showed that the ratio of $\mathrm{Bcl}-2 / \mathrm{Bax}$ in the treatment group was significantly increased compared with the exposure group and vehicle group (Fig. 7). Although the expression levels of Bax and Bcl-2 proteins showed no significant difference among groups after exposure, we found significant downregulation of Caspase- 3 in the treatment group. Therefore, we hypothesize that the high ratio of Bcl-2/Bax may be directly related to the release of Caspase-3, which eventually leads to apoptosis of lung tissue after exposure to silica. AD-MSC transplantation played an anti-apoptotic role in suppressing the downregulation of $\mathrm{Bcl}-2$ protein in rats after oral tracheal intubation with silica suspension. Accordingly, the inhibition of Caspase-3 on account of the decrease in the ratio of $\mathrm{Bax} / \mathrm{Bcl}-2$ can result in declined apoptosis in the treatment group. These findings further confirmed that AD-MSCs appear to exert pulmonary protection and reduce the process of apoptosis by inhibiting the expression of proteins related to the mitochondrial apoptotic pathway in the secondary damage of experimental silicosis in rats. Thus, the data from this study indicated that AD-MSCs can mitigate silica-induced lung fibrosis in rats. We also realized that this is preliminary work from the point of view of translation study and more work is required to establish the intervening and even therapeutic effects of AD-MSCs in silica-induced lung fibrosis. A study is underway to explore the effects of AD-MSC on an established silicosis model and compare their efficacy with BM-MSCs.

\section{Conclusions}

Transplanted AD-MSCs can significantly reduce the pulmonary inflammatory response induced by silica suspension in rats. AD-MSCs can also inhibit mitochondrial apoptosis-related protein expression, thereby inhibiting the process of silicosis. Although the apoptotic pathways require verification, we provide ample evidence for the further study of AD-MSCs in the treatment of silicosis. Our data suggest that AD-MSCs may have a beneficial effect on pulmonary fibrosis and may represent the basis of a new treatment for patients with silicosis. The successful development of stem cells in silicosis therapy will require a better understanding of host-graft interaction, the microenvironment and intrinsic characteristics, and subsequent greater functional improvement. Ongoing behavioral and biochemical assessment of long-term positive effects of $\mathrm{AD}-\mathrm{MSCs}$ will provide further insight regarding the therapeutic potential of AD-MSCs for silicosis.

\section{Abbreviations}

AD-MSC: Adipose-derived mesenchymal stem cell; ANOVA: One-way analysis of variance; BM-MSC: Bone marrow-derived mesenchymal stem cell;

DMEM: Dulbecco's modified Eagle's medium; H\&E: Hematoxylin and eosin; 
HGF: Hepatocyte growth factors; MSC: Mesenchymal stem cell; OD: Optical density; PBS: Phosphate buffered solution; SD: Sprague Dawley;

TUNEL: Terminal deoxynucleotide transferase (TdT)-mediated dUTP nick end labeling

\section{Acknowledgements}

The authors would like to express their gratitude to Dr Qiang Jia, Dr Gongchang Yu and Miss Yan Jiang for their assistance in performing the experiments.

\section{Funding}

This study was supported by grants from the National Natural Science Foundation of China (NSFC) (No. 81602893), Natural Science Foundation of Shandong Province (NSFSP) (No. ZR2015YL049), Medical and Health Technology Development Plan Project of Shandong Province (No. 2016WS0540), Key Research and Development Plan of Shandong Province (No. 2017GSF18186) and Innovation Project of Shandong Academy of Medical Science.

\section{Availability of data and materials}

The data that support the findings of this study are available from the corresponding author upon request.

\section{Authors' contributions}

SyC performed the experiments, analyzed and interpreted data, and drafted the manuscript. GqC, EgZ, YY, YjG and XyS performed the analysis and interpretation of data. CP participated in designing the experiment and preparing and writing the manuscript. MFL provided technical support for the analysis and critical revision of the manuscript. ZjD and HS designed the study and revised the manuscript. Authors read and approved the final manuscript.

\section{Ethics approval}

The animal procedures in this study were carried out in accordance with National Institutes of Health (NIH) Guidelines for the Care and Use of Laboratory Animals, with approval from the animal ethics committee of Shandong Academy of Medical Sciences.

\section{Consent for publication}

All authors of this manuscript agreed to publication.

\section{Competing interests}

The authors declare that they have no competing interests.

\section{Publisher's Note}

Springer Nature remains neutral with regard to jurisdictional claims in published maps and institutional affiliations.

\section{Author details \\ ${ }^{1}$ Department of Toxicology, Shandong Academy of Occupational Health and Occupational Medicine, Shandong Academy of Medical Sciences, Jinan, Shandong, People's Republic of China. '2Department of Respiratory Medicine, Qilu Children's Hospital of Shandong University, Jinan, Shandong, People's Republic of China. ${ }^{3}$ Queensland Alliance for Environmental Health Sciences (QAEHS), the University of Queensland, Brisbane, QLD, Australia. ${ }^{4}$ University of Queensland Centre for Clinical Research (UQCCR), the University of Queensland, Herston, Brisbane, QLD, Australia. ${ }^{5}$ School of Medicine and Life Sciences, University of Jinan-Shandong Academy of Medical Sciences, Jinan, Shandong, People's Republic of China. 'Department of Dermatology, Yueyang Hospital of Integrated Traditional Chinese and Western Medicine, Shanghai University of Traditional Chinese Medicine, Shanghai, People's Republic of China.}

\section{Received: 5 January 2018 Revised: 14 March 2018} Accepted: 16 March 2018 Published online: 19 April 2018

\section{References}

1. Rimal B, Greenberg AK, Rom WN. Basic pathogenetic mechanisms in silicosis: current understanding. Curr Opin Pulm Med. 2005;11:169-73.
2. Morikawa O, Walker TA, Nielsen LD, et al. Effect of adenovector-mediated gene transfer of keratinocyte growth factor on the proliferation of alveolar type II cells in vitro and in vivo. Am J Respir Cell Mol Biol. 2000;23:626-35.

3. Tzouvelekis A, Antoniadis A, Bouros D. Stem cell therapy in pulmonary fibrosis. Curr Opin Pulm Med. 2011;17:368-73.

4. Barczyk M, Schmidt M, Mattoli S. Stem cell-based therapy in idiopathic pulmonary fibrosis. Stem Cell Rev. 2015;11:598-620.

5. Zuk PA, Zhu M, Mizuno $H$, et al. Multilineage cells from human adipose tissue: implications for cell-based therapies. Tissue Eng. 2001;7:211-28.

6. Strem BM, Hicok KC, Zhu M, et al. Multipotential differentiation of adipose tissue-derived stem cells. Keio J Med. 2005;54:132-41.

7. Taghi GM, Ghasem KMH, Taghi L, et al. Characterization of in vitro cultured bone marrow and adipose tissue-derived mesenchymal stem cells and their ability to express neurotrophic factors. Cell Biol Int. 2012;36:1239-49.

8. Nakao N, Nakayama T, Yahata T, et al. Adipose tissue-derived mesenchymal stem cells facilitate hematopoiesis in vitro and in vivo: advantages over bone marrow-derived mesenchymal stem cells. Am J Pathol. 2010;177:547-54.

9. Liu WW, Wang HX, Yu W, et al. Treatment of silicosis with hepatocyte growth factor-modified autologous bone marrow stromal cells: a nonrandomized study with follow-up. Genet Mol Res. 2015;14:10672-81.

10. Liu K, Liu R, Cao G, et al. Adipose-derived stromal cell autologous transplantation ameliorates pulmonary arterial hypertension induced by shunt flow in rat models. Stem Cells Dev. 2011:20:1001-10.

11. Grainger SJ, Carrion B, Ceccarelli J, et al. Stromal cell identity influences the in vivo functionality of engineered capillary networks formed by co-delivery of endothelial cells and stromal cells. Tissue Eng Part A. 2013;19:1209-22.

12. Rojas $M, X u J$, Woods $C R$, et al. Bone marrow-derived mesenchymal stem cells in repair of the injured lung. Am J Respir Cell Mol Biol. 2005;33:145-52.

13. Shyamsundar M, Mc Auley DF, Ingram RJ, et al. Keratinocyte growth factor promotes epithelial survival and resolution in a human model of lung injury. Am J Respir Crit Care Med. 2014;189:1520-9.

14. Fang X, Neyrinck AP, Matthay MA, et al. Allogeneic human mesenchymal stem cells restore epithelial protein permeability in cultured human alveolar type II cells by secretion of angiopoietin-1. J Biol Chem. 2010;285:26211-22.

15. Prota LF, Lassance RM, Maron-Gutierrez T, et al. Bone marrow mononuclear cell therapy led to alveolar-capillary membrane repair, improving lung mechanics in endo-toxin-induced acute lung injury. Cell Transplant. 2010;19:965-71.

16. Weiss DJ, Bates JH, Gilbert T, et al. Stem cells and cell therapies in lung biology and diseases: conference report. Ann Am Thorac Soc. 2013;10:S25-44.

17. Curley GF, Hayes M, Ansari B, et al. Mesenchymal stem cells enhance recovery and repair following ventilator-induced lung injury in the rat. Thorax. 2012;67:496-501.

18. Ortiz LA, Gambelli F, Mc Bride C, et al. Mesenchymal stem cell engraftment in lung is enhanced in response to bleomycin exposure and ameliorates its fibrotic effects. Proc Natl Acad Sci U S A. 2003;100:8407-11.

19. D'Agostino B, Sullo $N$, Siniscalco $D$, et al. Mesenchymal stem cell therapy for the treatment of chronic obstructive pulmonary disease. Expert Opin Biol Ther. 2010;10:681-7.

20. Zhang WG, He L, Shi XM, et al. Regulation of transplanted mesenchymal stem cells by the lung progenitor niche in rats with chronic obstructive pulmonary disease. Respir Res. 2014;15(1):1-12.

21. Wang $H$, Yang YF, Zhao $L$, et al. Hepatocyte growth factor gene-modified mesenchymal stem cells reduce radiation-induced lung injury. Hum Gene Ther. 2013;24:343-53.

22. Jiang $H$, Zhang J, Zhang Z, et al. Effect of transplanted adipose-derived stem cells in mice exhibiting idiopathic pulmonary fibrosis. Mol Med Rep. 2015;12:5933-8

23. Rubio GA, Elliot SJ, Wikramanayake T, et al. Mesenchymal stromal cells prevent bleomycin-induced lung and skin fibrosis in aged mice and restore wound healing. J Cell Physiol. 2018. https://doi.org/10.1002/jcp.26418.

24. Baranowska A, Skowron B, Nowak B, et al. Changes in viability of rat adipose-derived stem cells isolated from abdominal/perinuclear adipose tissue stimulated with pulsed electromagnetic field. J Physiol Pharmacol. 2017;68:253-64

25. Hübner RH, Gitter W, El Mokhtari NE, et al. Standardized quantification of pulmonary fibrosis in histological samples. BioTechniques. 2008:44:507-11. 514-17

26. Zhao MM, Cui JZ, Cui Y, et al. Therapeutic effect of exogenous bone marrow-derived mesenchymal stem cell transplantation on silicosis via paracrine mechanisms in rats. Mol Med Rep. 2013;8:741-6. 
27. Gharaeekermani M, Gyetko MR, Hu B, et al. New insights into the pathogenesis and treatment of idiopathic pulmonary fibrosis: a potential role for stem cells in the lung parenchyma and implications for therapy. Pharm Res. 2007;24:819-41.

28. Lan YW, Theng SM, Huang TT, et al. Oncostatin M-preconditioned mesenchymal stem cells alleviate bleomycin-induced pulmonary fibrosis through paracrine effects of the hepatocyte growth factor. Stem Cells Transl Med. 2017;6:1006-17.

29. Wehner R, Wehrum D, Bornhäuser M, et al. Mesenchymal stem cells efficiently inhibit the proinflammatory properties of 6 -sulfo LacNAc dendritic cells. Haematologica. 2009:94:1151-6.

30. Barbarin $\mathrm{V}$, Xing Z, Delos $\mathrm{M}$, et al. Pulmonary overexpression of $\mathrm{IL}-10$ augments lung fibrosis and Th2 responses induced by silica particles. Am J Physiol Lung Cell Mol Physiol. 2005;288:L841-8.

31. Vitenberga Z, Pilmane M. Inflammatory, anti-inflammatory and regulatory cytokines in relatively healthy lung tissue as an essential part of the local immune system. Biomed Pap Med Fac Univ Palacky Olomouc Czech Repub. 2017;161:164-73

32. Li Y, Xiao J, Tan Y, et al. Inhibition of PKR ameliorates lipopolysaccharideinduced acute lung injury by suppressing NF-kB pathway in mice. Immunopharmacol Immunotoxicol. 2017;39:165-72.

33. O'Gorman MT, Jatoi NA, Lane SJ, et al. IL-1ß and TNF-a induce increased expression of CCL28 by airway epithelial cells via an NFkB-dependent pathway. Cell Immunol. 2005;238:87-96.

34. Agrawal S, Gollapudi S, Su H, et al. Leptin activates human B cells to secrete TNF-a, IL-6, and IL-10 via JAK2/STAT3 and p38MAPK/ERK1/2 signaling pathway. J Clin Immunol. 2011;31:472-8.

35. Zhang $X$, Yin $P$, Di D, et al. IL-6 regulates MMP-10 expression via JAK2/STAT3 signaling pathway in a human lung adenocarcinoma cell line. Anticancer Res. 2009;29:4497-501.

36. Liu CY, Liu YH, Lin SM, et al. Apoptotic neutrophils undergoing secondary necrosis induce human lung epithelial cell detachment. J Biomed Sci. 2003; 10:746-56.

37. Jernigan TW, Croce MA, Fabian TC. Apoptosis and necrosis in the development of acute lung injury after hemorrhagic shock. Am Surg. 2004; 70:1094-8.

38. Ashley $\mathrm{SL}$, Sisson $\mathrm{TH}$, Wheaton $\mathrm{AK}$, et al. Targeting inhibitor of apoptosis proteins protects from bleomycin-induced lung fibrosis. Am J Respir Cell Mol Biol. 2016:54:482-92.

39. Wang $X$, Zhou Y, Kim HP, et al. Hepatocyte growth factor protects against hypoxia/reoxygenation-induced apoptosis in endothelial cells. J Biol Chem. 2004;279:5237-43.

40. Marks N, Berg MJ, Guidotti A, et al. Activation of caspase-3 and apoptosis in cerebellar granule cells. J Neurosci Res. 2015;52:334-41.

\section{Submit your next manuscript to BioMed Central and we will help you at every step:}

- We accept pre-submission inquiries

- Our selector tool helps you to find the most relevant journal

- We provide round the clock customer support

- Convenient online submission

- Thorough peer review

- Inclusion in PubMed and all major indexing services

- Maximum visibility for your research

Submit your manuscript at www.biomedcentral.com/submit
Biomed Central 